\title{
LOW MASS STARS AND WHITE DWARFS IN NGC 6397
}

\author{
GUIDO DE MARCHI, FRANCESCO PARESCE \\ Space Telescope Science Institute \\ 3700 San Martin Drive, Baltimore MD 21218, USA \\ AND \\ MARTINO ROMANIELLO \\ Università di Pisa, Dipartimento di Fisica \\ Piazza Torricelli 2, I-56100 Pisa, Italy
}

Deep WFPC2 images in wide bands centered at 606 and $802 \mathrm{~nm}$ were taken with the HST 5.6 arcminutes from the center of the galactic globular cluster NGC 6397 . The images were used to accurately position $\sim 2120$ stars detected in the field on a color magnitude diagram down to a limiting magnitude $m_{814} \simeq m_{I} \simeq 26$ determined reliably and solely by counting statistics. A white dwarf sequence and a rich, narrow cluster main sequence are detected for the first time, the latter stretching from $m_{\mathbf{8 1 4}}=18.5$ to $m_{814}=24.0$ where it becomes indistinguishable from the field population. Two changes of slope of the main sequence at $m_{814} \simeq 20$ and $m_{814} \simeq 22.5$ are evident. The corresponding luminosity function increases slowly from $M_{814} \simeq 6.5$ to 8.5 as expected from ground based observations but then drops sharply from there down to the measurement limit. The corresponding mass function obtained by using the only presently available massluminosity function for the cluster's metallicity rises to a plateau between $\sim 0.25$ and $\sim 0.15 \mathrm{M}_{\odot}$, but drops towards the expected mass limit of the normal hydrogen burning main sequence at about $0.1 \mathrm{M}_{\odot}$. This result is in clear contrast to that obtained from the ground and implies either a substantial modification of the cluster's initial mass function due to dynamical evolution in its lifetime, or that very low mass stars are not produced in any dynamically significant amount by clusters of this type. The white dwarf sequence is in reasonable agreement with a cooling sequence of models of mass $0.5 \mathrm{M}_{\odot}$ at the canonical distance of NGC 6397 with a scatter that is most likely due to photometric errors, but may also reflect real differences in mass or chemical composition. 